and miniature motors. Large areas of TPC or multiple TPC plates can be employed to produce much higher electrical energy to power heavier systems.

This highly miniaturized, high-temperature, long-life power source can be supplementary to primary high-temperature battery. The concept applies famil- iar thermionic emission principle for power generation by harnessing the local heat in the application environments. The approach of power production and design flexibility naturally provides an attractive option to harness in situ heat to produce power enough to operate electronics and miniature instrumentation.
TPC can be designed to support geothermal explorations by harnessing heat energy of the local environment.

This work was done by Harish Manohara, Mohammad Mojarradi, and Harold F. Greer of Caltech for NASA's Jet Propulsion Laboratory. Further information is contained in a TSP (see page 1). NPO-46967

\title{
Graph Theory Roots of Spatial Operators for Kinematics and Dynamics
}

These concepts can be applied to modeling, simulation, and control of robots and other mechanisms.

\section{NASA's Jet Propulsion Laboratory, Pasadena, California}

Spatial operators have been used to analyze the dynamics of robotic multibody systems and to develop novel computational dynamics algorithms. Mass matrix factorization, inversion, diagonalization, and linearization are among several new insights obtained using such operators. While initially developed for serial rigid body manipulators, the spatial operators - and the related mathematical analysis - have been shown to extend very broadly including to tree and closed topology systems, to systems with flexible joints, links, etc. This work uses concepts from graph theory to explore the mathematical foundations of spatial operators. The goal is to study and characterize the properties of the spatial operators at an abstract level so that they can be applied to a broader range of dynamics problems.

The rich mathematical properties of the kinematics and dynamics of robotic multibody systems has been an area of strong research interest for several decades. These properties are important to understand the inherent physical behavior of systems, for stability and control analysis, for the development of computational algorithms, and for model development of faithful models.

Recurring patterns in spatial operators leads one to ask the more abstract question about the properties and characteristics of spatial operators that make them so broadly applicable. The idea is to step back from the specific application systems, and understand more deeply the generic requirements and properties of spatial operators, so that the insights and techniques are readily available across different kinematics and dynamics problems.

In this work, techniques from graph theory were used to explore the abstract basis for the spatial operators. The close relationship between the mathematical properties of adjacency matrices for graphs and those of spatial operators and their kernels were established. The connections hold across very basic requirements on the system topology, the nature of the component bodies, the indexing schemes, etc. The relationship of the underlying structure is intimately connected with efficient, recursive computational algorithms. The results provide the foundational groundwork for a much broader look at the key problems in kinematics and dynamics.

The properties of general graphs and trees of nodes and edge were examined, as well as the properties of adjacency matrices that are used to describe graph connectivity. The nilpotency property of such matrices for directed trees was reviewed, and the adjacency matrices were generalized to the notion of block weighted adjacency matrices that support block matrix elements. This leads us to the development of the notion of Spatial Kernel Operator SKO kernels. These kernels provide the basis for the development of SKO resolvent operators.

This work was done by Abhinandan Jain of Caltech for NASA's Jet Propulsion Laboratory. For more information, contact iaoffice@jpl.nasa.gov. NPO-47313

\section{* Spacesuit Soft Upper Torso Sizing Systems}

This system has application in medical devices for immobilizing injured limbs or applying controlled pressure to areas of the body of a burn victim.

\section{Lyndon B. Johnson Space Center, Houston, Texas}

The passive sizing system consists of a series of low-profile pulleys attached to the front and back of the shoulder bearings on a spacesuit soft upper torso (SUT), textile cord or stainless steel cable, and a modified commercial ratchet mechanism. The cord/cable is routed through the pulleys and attached to the ratchet mechanism mounted on the front of the spacesuit within reach of the suited subject. Upon actuating the ratchet mechanism, the shoulder bearing breadth is changed, providing variable upper torso sizing.

The active system consists of a series of pressurizable nastic cells embedded into the fabric layers of a spacesuit SUT. These cells are integrated to the front and back of the SUT and are connected to an air source with a variable regulator. When inflated, the nastic cells provide a change in the overall shoulder bearing breadth of the spacesuit and thus, torso sizing.

The research focused on the development of a high-performance sizing and actuation system. This technology has application as a suit-sizing mechanism to 